Psychotherapeut 2002 $\cdot 47: 240$ DOI 10.1007/s00278-002-0240-y

\section{Redaktion \\ M. Cierpka, Heidelberg}

Unter ständiger Mitarbeit

M. Stasch, Heidelberg
Psychotherapie im Internet

Elisabeth Althaus • Düsseldorf mmer wieder taucht der ZappelPhilipp als typisches Beispiel auf: das hyperaktive Kind setzt nicht nur die Eltern unter einen massiven Leidensdruck, auch die Kinder selbst haben mit enormen Schwierigkeiten zu kämpfen. Nachdem klinische Daten die Wirksamkeit des Amphetamins Methylphenidat (Ritalin ${ }^{\circledR}$ ) bei der Therapie des Hyperaktivitätssyndroms gezeigt haben (http://consensus.nih.gov/cons/110/110_st atement.htm), verwundern daher die stark gestiegenen Verordnungszahlen nicht. So hat die Drogenbeauftragte der Bundesregierung festgestellt, dass die Verwendung von Methylphenidat von 1993 bis 2001 um das 2ofache gestiegen ist (http://www.bmgesundheit.de/themen/drogen/pm/260402.htm). Fehlverordnungen, missbräuchliche Verwendung seien damit nicht ausgeschlossen. Sie fordert daher eine multimodale Therapie, wie sie auch die Leitlinien der Deutschen Gesellschaft für Kinder- und Jugendpsychiatrie und -psychotherapie beschreiben (http://www.uni-duesseldorf.de/WWW/AWMF/ll/kjpp-o19.htm). Diese empfehlen eine Kombination aus Familientherapie, kognitiver Therapie des Kindes, Pharmakotherapie sowie evtl. einer oligoantigenen Diät. Ähnlich sind die Therapieempfehlungen aus kinderärztlicher Sicht, die unter http://www. ag-adhs.de/public/ag-adhs/leitlinie.html nachzulesen sind.

Als jüngste Konsequenz aus der Diskussion um Ritalin ${ }^{\circledR}$ hat eine parteiübergreifende Gruppe von 118 Abgeordneten am 07. Mai 2002 einen Antrag an die Bundesregierung gestellt, dass „Diagnose und Therapie des Aufmerksamkeitsdefizits- und Hyperaktivitätssyndroms (ADHS) nur noch von hierfür qualifizierten Ärzten vorgenommen werden" sollen. Insbesondere soll auch die Erforschung der Langzeitfolgen einer Einnahme von Methylphenidat vorangetrieben werden (http://www.

\title{
Hilfe für den Zappel-Philipp: Das Hyperaktivitätssyndrom
}

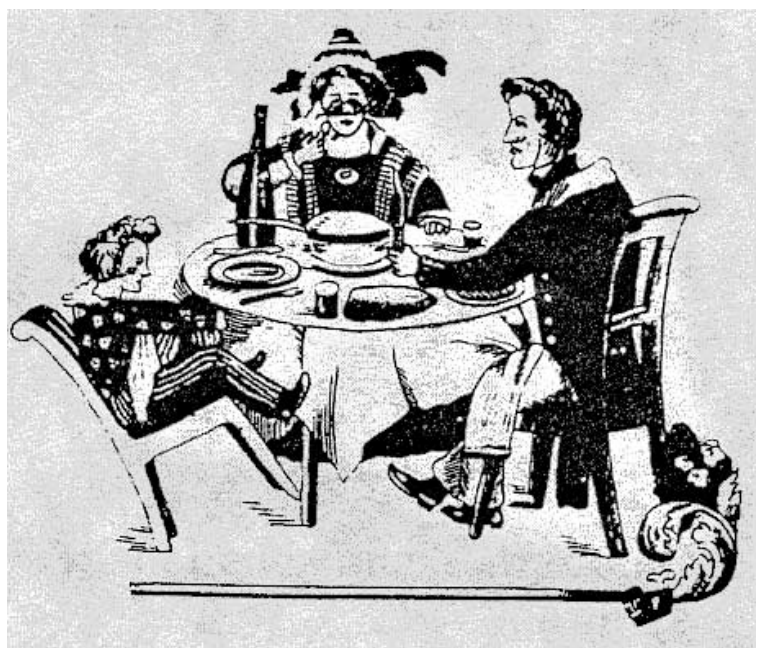

Abb. 1 Der Zappel-Philipp als Beispiel eines hyperaktiven Kindes (http://www. groeller.de) bundestag.de/aktuell/hib/2002_116/o3. html). Die Debatte in Ausschüssen und Plenum steht noch aus.

Doch welche Alternativen gibt es zu Ritalin ${ }^{\circledR}$ ? Ein auf der Familientherapie basierender ganzheitlicher Ansatz wird unter http://www.systemisch.net dargestellt. Dr. Ingo Spitzok von Brisinsky beschreibt hier ausgewählte Konzepte der systemischen Therapie bei Hyperaktivitätssyndrom. Aus der Sicht der Sozialpädagogin Dr. Beate Gröller bietet ein verändertes Interaktionsverhalten eine Chance für hyperaktive Kinder. Sie stellt unter http://www.groeller.de einen Trainingskurs zur Veränderung von Einstellungen vor (Abb. 1). Geänderte Einstellung und Wahrnehmung der Eltern führen $\mathrm{zu}$ veränderten Interaktionen, zum Akzeptieren des Kindes. Kindzentrierte Therapien ergänzen dieses Behandlungskonzept.

Wissenschaftlich abgestützte Informationen bietet ADD-Online unter http://www.psychologie-online.ch/add/ mit Hinweisen zur Diagnostik und Therapie bei Erwachsenen, Kindern und Jugendlichen mit Attention Deficit Disorder. Diskussionsforen und der Zugang zu einer geschlossenen FachMailingliste fördern den Erfahrungs- austausch. Alle wesentlichen Links zum Bereich Aufmerksamkeitsdefizitsyndrom/hyperkinetisches Syndrom finden sich unter http://www.kinderpsychiater.org/linksads.htm. Dort steht auch ein Hinweis auf http.//www.hyperaktiv.de. Unter der Rubrik Forschung sind hier wesentliche Arbeiten zur Genetik, Diagnostik und Neurobiologie sowie ein Übersichtsartikel zur Therapie nachzulesen. Informationen über Selbsthilfegruppen, Telefonberatungsstellen und Veranstaltungen für Betroffene und Angehörige erhält man beim Bundesverband Arbeitskreis Überaktives Kind e.V.unter http://www.auek.de. Hier wird auch die ADHD-Profil-Studie vorgestellt, die in einer bundesweiten Fragebogenaktion typische Entwicklungsverläufe, Risikofaktoren, Therapieerfolge und soziale Auswirkungen erfassen soll. Vielleicht trägt diese Studie unter Zusammenarbeit mit dem Charité/Virchow-Klinikum Berlin dazu bei, die Versorgung von hyperaktiven Kindern in Deutschland zu verbessern.

(C) Springer-Verlag 2002

Elisabeth Althaus

Karolingerstraße 118,40223 Düsseldorf E-Mail:EAAlthaus@gmx.de 